Check for updates

Cite this: RSC Adv., 2018, 8, 18347

Received 5th April 2018

Accepted 11th May 2018

DOI: $10.1039 / c 8 r a 02930 j$

rsc.li/rsc-advances

\title{
Free-standing polydopamine films generated in the presence of different metallic ions: the comparison of reaction process and film properties $\dagger$
}

\begin{abstract}
Xuwen Han, Feng Tang and Zhaoxia Jin (D)*
Polydopamine is widely used in surface modification, nanofiltration, photonic devices and drug delivery. The formation mechanism and properties of polydopamine are modified by the experimental conditions. Herein we demonstrated a comparison study of free-standing polydopamine films generated at the air-solution interface and their corresponding nanoparticles in solutions, in the presence of various metallic cations, $\mathrm{Na}^{+}, \mathrm{Ca}^{2+}, \mathrm{Mg}^{2+}$ and $\mathrm{Co}^{2+}$. Adding metallic ions influenced the intermediates in dopamine polymerization, and in turn the morphology and properties of the produced free-standing polydopamine films. Moreover, we observed that the polymerization process accompanying the stratification determines the formation of free-standing films at the air-solution interface: the fast polymerization of dopamine in a $\mathrm{Co}^{2+}$ environment leads to a rugged film surface and porous film body, whereas the comparatively slow polymerization of dopamine under conditions of other metallic ions results in a smooth and solid film. In addition, the water contact angles of the upper and lower surface of the polydopamine films were different. This investigation enriches our knowledge of dopamine polymerization in different environments, which is particularly useful for further application of freestanding polydopamine films.
\end{abstract}

\section{Introduction}

The formation of free-standing polydopamine (PDA) films has attracted great attention recently. ${ }^{1-9}$ PDA films show different colours modulated by the polymerization conditions, ${ }^{3}$ and they can be transferred to various substrates to form functional coatings. A promising humidity sensor with quick response and recovery time was developed based on PDA films. ${ }^{4}$ For widening the application of PDA films, the knowledge of their formation mechanism and the manipulation of their properties via controlling the polymerization process are urgently required. ${ }^{10}$

Free-standing PDA film can be formed at the air-water interface. ${ }^{2,3}$ Accompanying it, PDA nanoparticles are also generated in the solution phase. Researchers generally focused

Department of Chemistry, Renmin University of China, Beijing, 100872, People's Republic of China. E-mail: jinzx@ruc.edu.cn

$\dagger$ Electronic supplementary information (ESI) available: Fig. S1-S10 and Tables S1-S5 (PDF). UV-vis spectra of oxidative polymerization of dopamine and dopamine in the existence of $\mathrm{Co}^{2+}$ metal ions; SEM images of Mg-PDA film formation at $60{ }^{\circ} \mathrm{C}$ for 2 days; AFM images of PDA films for 150 minutes reaction; SEM images of PDA films for 20 days reaction; the high-resolution XPS spectra of the C 1s, N 1s and O 1s for PDA films; Raman spectra of the lower surfaces of PDA films; DLS of PDA nanoparticles after $1 \mathrm{~h}$ reaction. The purity and the contents of main impurity in four salts. The contents of different metallic elements in PDA films and corresponding nanoparticles obtained by ICP-OES. The detailed component in $\mathrm{C} 1 \mathrm{~s} \mathrm{O}$ 1s and $\mathrm{N}$ 1s of polydopamine films and nanoparticles generated in different conditions based on XPS. See DOI: $10.1039 / \mathrm{c} 8 \mathrm{ra02930j}$ either on PDA nanoparticles or on the films. ${ }^{2}$ But a comparison study of the properties of PDA films and nanoparticles may present their correlation and difference. It will help us in understanding the environmental influence on PDA and elucidating the formation mechanism of free-standing PDA films. On the other hand, it is well documented that metallic-ionbased crosslinking of biopolymers contributes strongly to mechanically enhanced biomaterials. ${ }^{11-14}$ The cuticle of mussel byssal threads is generated in salty sea water. ${ }^{11,15,16}$ The coordination interactions between $\mathrm{Fe}^{3+}$ and catechol-bearing proteins play important roles in the modulation of chemical structure and mechanical strength of mussel byssal threads. ${ }^{17-20}$ However, systematic knowledge of the effect of different metallic cations involved in the oxidative polymerization of dopamine is still lacking. For instance, although the concentrations of $\mathrm{Na}^{+}, \mathrm{Ca}^{2+}$ and $\mathrm{Mg}^{2+}$ ions are much higher than that of other metallic ions in the natural environment (sea water), a comparison study of their effects on the oxidative polymerization of dopamine and their influence on the properties of PDA films is so far unexplored. In a previous study, Klosterman and Bettinger first observed that the properties of PDA films have been changed after post-incubation in solutions containing redox-inactive metal cations $\left(\mathrm{NaCl}, \mathrm{MgCl}_{2}\right.$ and $\left.\mathrm{CaCl}_{2}\right){ }^{21}$ So these metallic cations would have a more pronounced influence on the properties of PDA if they were added in the oxidative polymerization of dopamine. 
Herein, we demonstrated a comprehensive investigation of the oxidative polymerization of dopamine in the presence of four metallic cations, $\mathrm{Na}^{+}, \mathrm{Ca}^{2+}, \mathrm{Mg}^{2+}$ and $\mathrm{Co}^{2+}$, respectively, about both the free-standing films and the nanoparticles. The comparison of morphologies and chemical compositions of obtained free-standing PDA films and corresponding nanoparticles was conducted to elucidate their correlation and difference. Based on these characterizations, different intermediates in formation of PDA films in various metallic ions were proposed. Moreover, we noticed that the water contact angle of upper and lower surface of these PDA films were different, that is the lower surface (exposed to solution, similar with nanoparticles in property) was more hydrophilic than the upper surface.

\section{Experimental sections}

\section{Materials}

Dopamine hydrochloride (purity 98\%) was purchased from Sigma-Aldrich. Sodium chloride ( $\mathrm{NaCl}, \mathrm{AR}$ ) was purchased from Beijing Chemical Works. Cobalt chloride hexahydrate $\left(\mathrm{CoCl}_{2}-\right.$ $\cdot 6 \mathrm{H}_{2} \mathrm{O}, \mathrm{CP}$ ) was obtained from Shanghai Macklin Biochemical Co. Ltd. Magnesium chloride hexahydrate $\left(\mathrm{MgCl}_{2} \cdot 6 \mathrm{H}_{2} \mathrm{O}, \mathrm{AR}\right)$ and calcium chloride anhydrous $\left(\mathrm{CaCl}_{2}, \mathrm{AR}\right)$ were purchased from Sinopharm Chemical Reagent Co. Ltd. The purity and the contents of main impurity in these four salts were shown in Table S1. $\uparrow$ Tris(hydroxymethyl)aminomethane (Tris)-HCl buffer $(\mathrm{pH}=8.8)$ was obtained from Shanghai Double-Helix Biotech. Co. Ltd. All these reagents were used as received. Water was purified (18.2 M $\Omega \mathrm{cm}$ ) using Mingche ${ }^{\mathrm{TM}}-\mathrm{D} 24 \mathrm{UV}$ system (Merck Millipore).

\section{Fabrication of free-standing PDA films and nanoparticles}

In a typical experiment, PDA film was prepared by dissolving dopamine hydrochloride $(10 \mathrm{mM})$ in water $(10 \mathrm{~mL})$ in a plastic Petri dish, and then Tris-buffer $(\mathrm{pH}=8.8,10 \mathrm{mM})$ was added to above solution. The solution was kept stable in ambient air for different time. Petri dishes were covered to avoid the falling dust and fast evaporation of water. PDA film appeared at the air-water interface after $50 \mathrm{~min}$ at room temperature $\left(20{ }^{\circ} \mathrm{C}\right)$. After desired reaction time, the oxidative polymerization of dopamine was stopped. The films floating on the solution's surface and the dark suspension were washed with de-ionized water several times separately to remove loosely-bond chemicals. The films or the nanoparticles were recognized as clean one when there was clear in its washing solution $(1 \mathrm{~mL})$ if dripping $\mathrm{AgNO}_{3}(0.1 \mathrm{mM}, 1 \mathrm{~mL})$ aqueous solution. Then clean PDA films and nanoparticles were collected separately and dried by freeze-drying $\left(-58{ }^{\circ} \mathrm{C}, 5-6 \mathrm{~Pa}\right)$. The dried samples were used to conduct morphological and chemical characterizations.

For preparing PDA $(\mathrm{Na} / \mathrm{Mg} / \mathrm{Ca} / \mathrm{Co})$ films, $100 \mathrm{mM}$ salt $(\mathrm{NaCl}$ $58.44 \mathrm{mg}, \mathrm{MgCl}_{2} \cdot 6 \mathrm{H}_{2} \mathrm{O} 203.30 \mathrm{mg}, \mathrm{CaCl}_{2} 110.98 \mathrm{mg}, \mathrm{CoCl}_{2}-$ $\left.\cdot 6 \mathrm{H}_{2} \mathrm{O} 237.93 \mathrm{mg}\right)$ was dissolved in water $(10 \mathrm{~mL})$ in a plastic Petri dish. Then dopamine hydrochloride $(10 \mathrm{mM})$ and Trisbuffer ( $\mathrm{pH}=8.8,10 \mathrm{mM}$ ) were added to above solution. These solutions were kept stable for different time. After desired reaction time, the obtained films floating on solution surface and the suspension in solutions were washed with de-ionized water separately and dried by freeze-drying.

\section{Characterizations of PDA films and PDA nanoparticles}

The morphologies of these samples were observed by scanning electron microscopy (SEM, Hitachi, SU8010) and atomic force microscope (AFM, Burker, Dimension ICON). The films were directly pasted on the conductive adhesive. Samples were coated with a thin layer of gold before SEM characterization. SEM was performed at an accelerating voltage of $4 \mathrm{kV}$. AFM images were obtained using a ScanAsyst-Air probe operated in ScanAsyst mode. Silicon wafers were cleaned by sonication in acetone, followed by isopropyl alcohol and then rinsed with deionized water. PDA films generated after different reaction times were transferred on silicon substrate $(1.5 \mathrm{~cm} \times 1.5 \mathrm{~cm})$ and then washed by de-ionized water, and dried in vacuum oven at $30{ }^{\circ} \mathrm{C}$ overnight before AFM characterizations.

Ultraviolet-visible (UV-vis) absorption was measured using a Varian Cary 50 UV-vis spectrophotometer. The spectra of abovementioned reaction solutions were recorded every 1-2 minutes for several hours by taking $1 \mathrm{~mL}$ solution diluted in $2 \mathrm{~mL}$ de-ionized water. Zeta potentials and particle sizes of clean PDA nanoparticles were recorded at different $\mathrm{pH}$ conditions by using a Malvern Nano-ZS90 ZETASIZER at $25^{\circ} \mathrm{C}$. Water contact angle measurements for various PDA films were performed by using an optical contact angle apparatus $(4 \mu \mathrm{L}$ droplet) (KRÜ SS drop shape analyzer DSA30). Tested PDA films were attached on plastic Petri dishes and dried in vacuum oven at $30{ }^{\circ} \mathrm{C}$ overnight before measurement. Each data was the average of five measurements.

X-ray photoelectron spectroscopy (XPS) was performed on a Imaging Photoelectron Spectrometer (Kratos Analytical Ltd., Axis Ultra) with monochromatic $\mathrm{Al} \mathrm{K} \alpha(h \nu=1486.7 \mathrm{eV})$ radiation as the excitation and an X-ray power of $150 \mathrm{~W}$. All spectra were calibrated using the hydrocarbon $\mathrm{C}$ 1s peak $(284.8 \mathrm{eV})$. Highresolution scans were acquired for $\mathrm{C} 1 \mathrm{~s}, \mathrm{~N} 1 \mathrm{~s}$, $\mathrm{O} 1 \mathrm{~s}$ regions. The peak-fitting procedure was consistent with the best fit with consideration of peak position, full width at a half-maximum and intensity. The contents of metallic elements in PDA films and nanoparticles were taken out by using Inductively Coupled Plasma Optical Emission Spectrometry (ICP-OES, Agilent, ICP/ 700). Before ICP-OES characterizations, all samples (ca. 1.2 mg) were totally decomposed in $\mathrm{KOH}(\mathrm{KOH}, 8 \mathrm{M})$ aqueous solution with the assistance of $\mathrm{H}_{2} \mathrm{O}_{2}$ under sonication. For avoiding the impurity brought by $\mathrm{KOH}$ solution in which other metallic ions may exist as impurities, the metallic elements in $\mathrm{KOH}$ solution was also measured and deducted as background in calculation.

\section{Results and discussion}

\section{The characterization of reaction process and film morphologies}

The presence of different types of metallic ions showed distinct impact on the polymerization of dopamine. Adding Tris buffer 
in the mixture of dopamine and metallic ions solution triggered the oxidative polymerization of dopamine, but the reactions were separated with each other by the different colour change (Fig. 1). The addition of sodium ions showed no influence on the speed of oxidative polymerization of dopamine, calcium and magnesium ions slightly accelerated it, while the reaction in cobalt(II) ions significantly speeded up. In the system with magnesium(II), the violet colour appeared in the solution during the oxidative polymerization, distinguishing it from other systems. We tracked the reaction process of these systems by UV-vis spectroscopy.

Fig. 2 presented consecutively recorded UV-vis spectra of dopamine polymerization in the presence of $\mathrm{Na}^{+}, \mathrm{Ca}^{2+}, \mathrm{Mg}^{2+}$ and $\mathrm{Co}^{2+}$ cations. UV-vis spectra of neat dopamine system were recorded as a contrast (Fig. $\mathrm{S} 1 \dagger$ ). In the case of $\mathrm{Co}^{2+}$, because the absorption of $\mathrm{Co}^{2+}$ ions (Fig. 2d), to clearly identify the change of these spectra, the absorption spectrum of $\mathrm{Co}^{2+}$ was subtracted as shown in Fig. S2. $\dagger$ After adding Tris for $1 \mathrm{~min}$, the spectra of all systems presented a broad absorption at $450 \mathrm{~nm}$ (red line). Then this absorption separated to distinct peaks in different conditions. Taken the green lines in four systems for a comparison, the peak at $480 \mathrm{~nm}$ in all spectra is belonged to the aminochrome (AC) based on literatures; ${ }^{22}$ while the peak at $410 \mathrm{~nm}$ in $\mathrm{Na}^{+}$and $413 \mathrm{~nm}$ in $\mathrm{Ca}^{2+}$, similar to that in pure dopamine case (Fig. $\mathrm{S} 1 \dagger$ ), could be assigned to the formation of yellow chromophore dopamine- $o$-quinone (DQ). ${ }^{22}$ In the difference spectra of $\mathrm{Co}^{2+}$ system (Fig. S2†), there was only a peak around $480 \mathrm{~nm}$ (belongs to $\mathrm{AC}$ ) observed in the first several minutes of oxidative polymerization of dopamine. Bisaglia et al. have noticed that when the oxidative polymerization of dopamine happened in a physiological conditions, i.e., at $37^{\circ} \mathrm{C}$ and pH 7.4 (phosphate buffer), the peaks corresponding to AC were

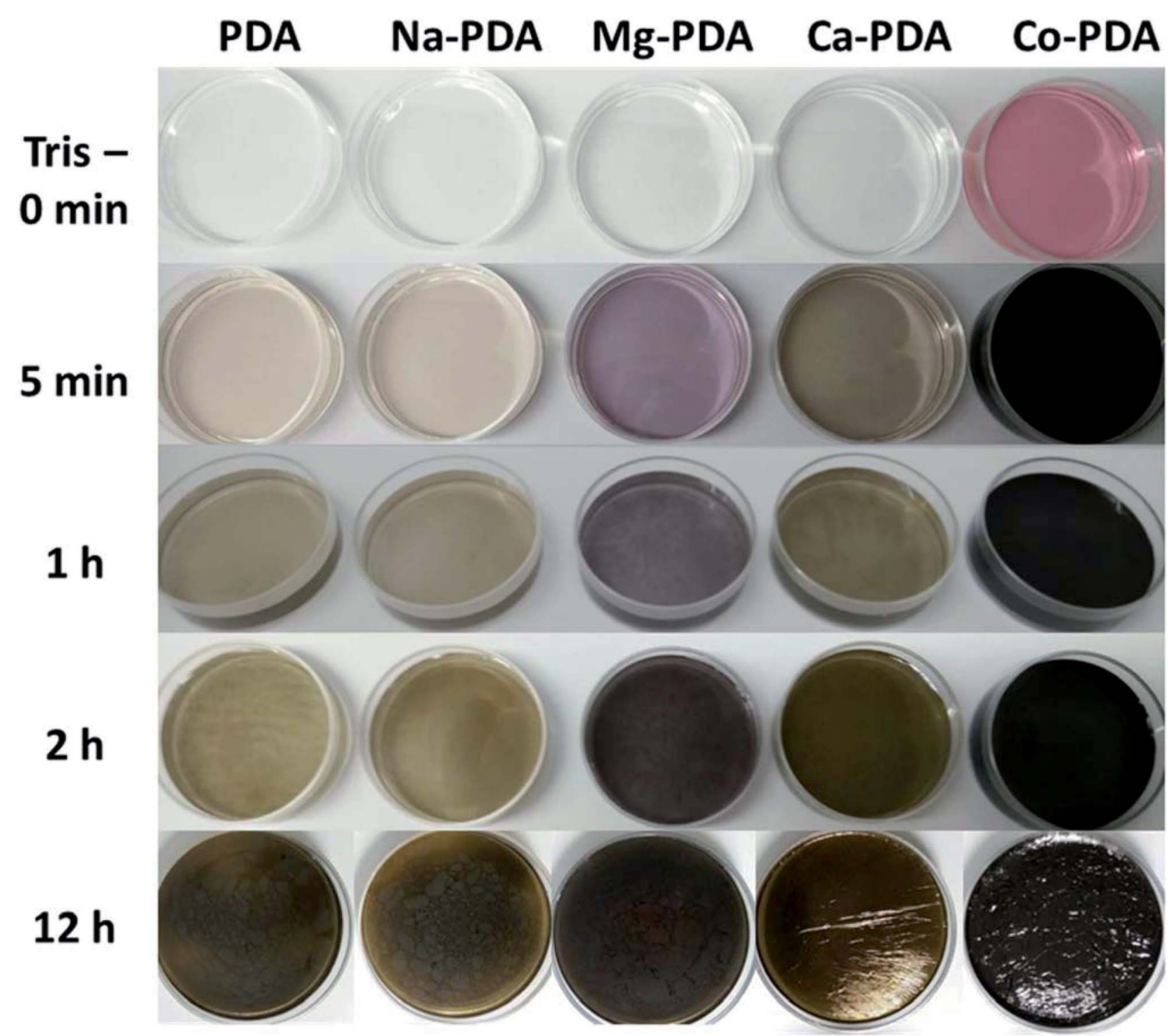

Fig. 1 Images of PDA films formation in the presence of different metallic ions after adding Tris buffer. The concentration of dopamine was $10 \mathrm{mM}$, the concentrations for various metallic ions were $100 \mathrm{mM}$. The concentration of Tris buffer was $10 \mathrm{mM}, \mathrm{pH}=8.8$. 

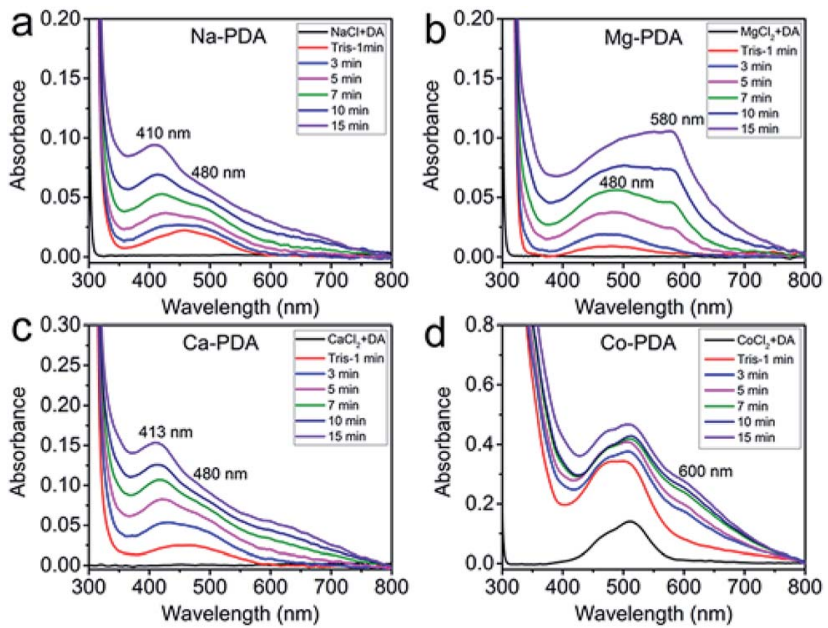

Fig. 2 Consecutively measured UV-vis absorption spectra of the oxidative polymerization of dopamine in the presence of different salts. (a) $\mathrm{Na}^{+}(100 \mathrm{mM}),(b) \mathrm{Mg}^{2+}(100 \mathrm{mM}),(\mathrm{c}) \mathrm{Ca}^{2+}(100 \mathrm{mM}),(\mathrm{d}) \mathrm{Co}^{2+}$ (100 mM).

the only observed in the initial stage. ${ }^{22}$ Because of the fast increase of PDA nanoparticle size in $\mathrm{Co}^{2+}$ case, scatteringinduced broad absorption around $600 \mathrm{~nm}$ increased gradually. However, in $\mathrm{Mg}^{2+}$ case, the peak of AC merged with the absorption peak at $580 \mathrm{~nm}$, indicating new intermediate species in oxidative polymerization of dopamine in $\mathrm{Mg}^{2+}$ ions conditions. It can be assigned to the quinone dimer based on literatures. ${ }^{23}$ The unique purple color in Mg-dopamine may be due to the appearance of this special intermediate.

Another interesting phenomenon is the PDA films obtained in different environments shone different colours. Ca-PDA film reflects blue-green colour, and Mg-PDA film likes shiny mirror, while others are black. It is reported that the colour of PDA films related with the films' morphology and thickness. ${ }^{3}$ Because we have maintained the concentrations of dopamine, metallic ions and Tris buffer at same in different systems, we thought the difference of these systems in thickness or morphology demonstrated the different influence of metallic ions on the oxidative polymerization of dopamine. Fig. 3 presented SEM images of all PDA films (obtained after 2 days reaction) generated in different conditions, their upper surfaces, lower surfaces and cross-sections. The upper surfaces of all PDA films were smooth like skin, while there were PDA nanoparticles with different sizes on their lower surfaces. Isolated particles shown on Na-PDA, Mg-PDA and Ca-PDA films were similar with that on pure PDA films, but those on Co-PDA films were disorderlypacked large aggregation of nanoparticles. The film thickness was obtained by measuring the thickness of skin-layer of broken PDA films (Fig. 3, cross-section images of PDA films). Except CoPDA film that was too rugged and without clear skin-layer, the skin's thickness of Na-PDA (about $145 \mathrm{~nm}$ ) was near that of neat PDA (144 nm), while it was $195 \mathrm{~nm}$ for Mg-PDA film and $270 \mathrm{~nm}$ for Ca-PDA film, respectively. The shiny colour of Mg-PDA and Ca-PDA films may be due to their increased thickness compared with that of PDA and Na-PDA films, and densely packed film body. To further confirm the origin of the shiny colour, the MgPDA film was fabricated at a higher temperature $\left(60^{\circ} \mathrm{C}\right)$. It became rugged and was not reflective to light (Fig. S3†). So these different colours indeed originated from the differences in thickness and morphologies of these PDA films generated in the presence of different ions.

To identify the details of PDA films in their initial state, AFM characterizations of PDA films at different reaction times have been conducted. Fig. 4 demonstrated the upper surface morphologies of PDA films floated on the air-solution surface after $1 \mathrm{~h}$ reaction in different conditions. The RMS of neat PDA, Na-PDA, Mg-PDA was less than $1 \mathrm{~nm}$, that of Ca-PDA films was $\sim 1.5 \mathrm{~nm}$, but that of Co-PDA film was much higher than abovementioned samples, about $7.5 \mathrm{~nm}$. Although the size of PDA nanoparticles slightly increased with increasing reaction time (150 min), the roughness of these PDA films was similar with that samples taken after reaction for $1 \mathrm{~h}$ (Fig. S4 $\dagger$ ). The elongation of reaction time increases the thickness of all films. We have kept the reaction as long as 20 days and we found that the

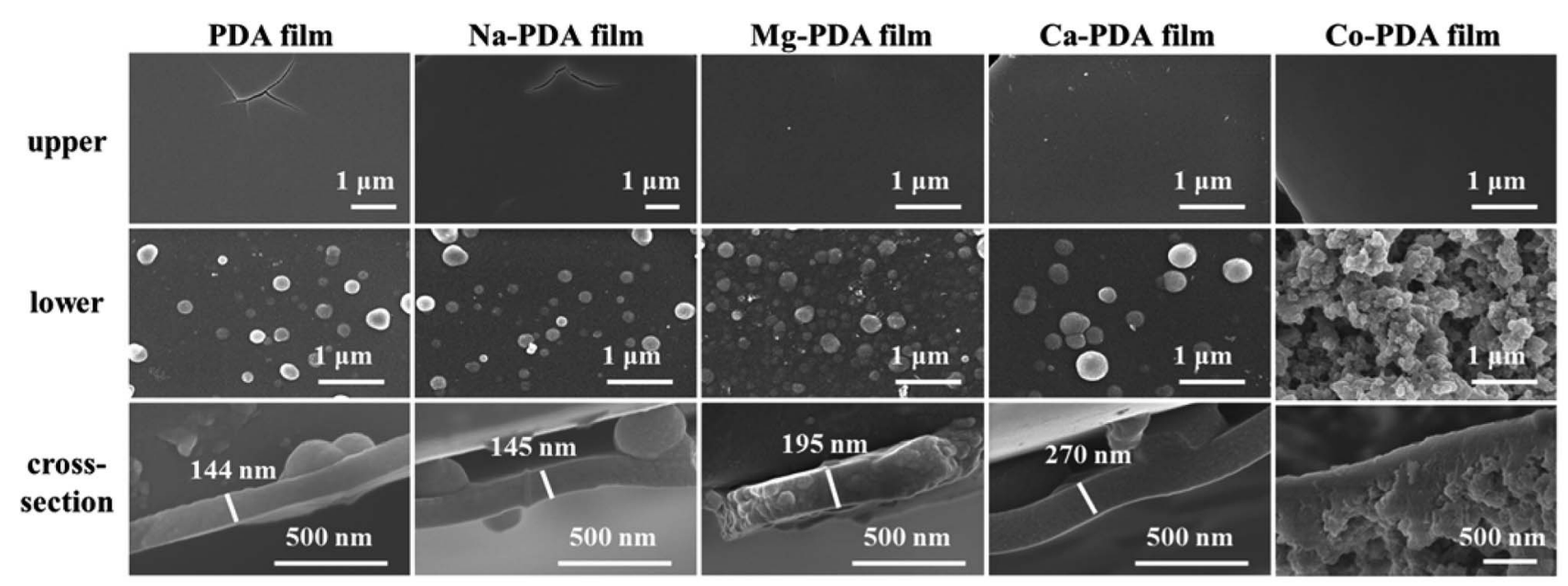

Fig. 3 SEM images of PDA films generated in the presence of different metal ions, compared with pure PDA. Pictures on first line: upper surfaces exposed to air. Pictures on second line: lower surfaces exposed to solutions. Pictures on third line: cross-sections of films shown their thickness. All PDA films were obtained after 2 days reaction. 
a

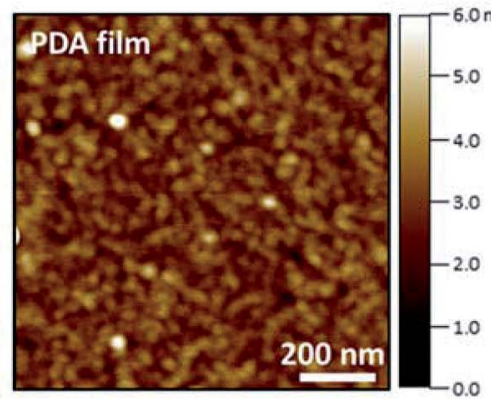

d

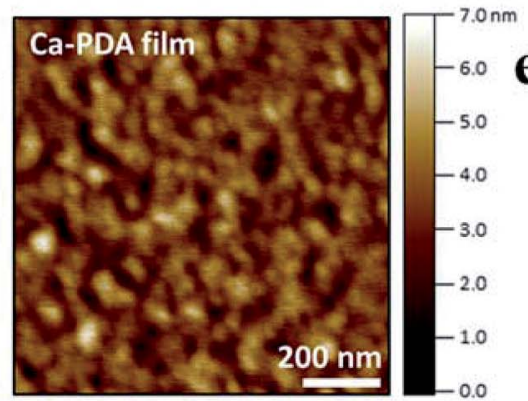

\section{b}
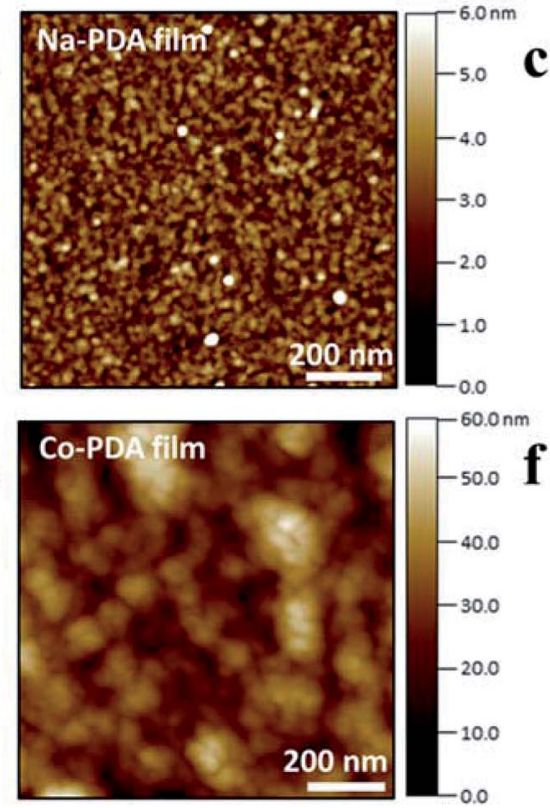

c

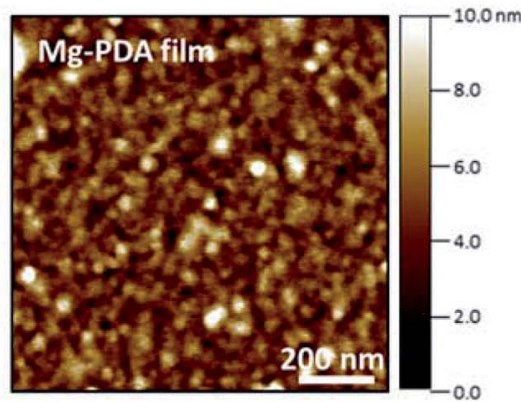

\section{f}

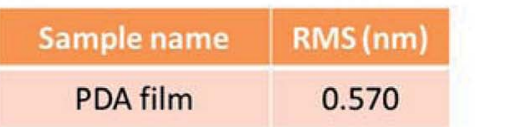

\begin{tabular}{|l|l|}
\hline Na-PDA film & 0.796 \\
\hline Mg-PDA film & 1.420 \\
\hline Ca-PDA film & 0.818 \\
\hline Co-PDA film & 7.490 \\
\hline
\end{tabular}

Fig. 4 AFM images of upper surface of PDA films generated in the presence of different metallic ions after $1 \mathrm{~h}$ reaction. (a) PDA, (b) Na-PDA, (c) Mg-PDA, (d) Ca-PDA, (e) Co-PDA. Analyses were performed in air at $20^{\circ} \mathrm{C}$ on $1 \mu \mathrm{m}^{2}$ areas. The RMS of every film was shown in (f).

thickness of Co-PDA was about $800 \mathrm{~nm}$, it was $500 \mathrm{~nm}$ for CaPDA film, $400 \mathrm{~nm}$ for pristine PDA, Na-PDA and Mg-PDA films (Fig. S5†). The growth of film thickness approached its limit in several days, the same as polydopamine film deposited on solid substrates.

The characterization of reaction process and film morphologies characterizations of chemical compositions of PDA films and nanoparticles

Table 1 presented the elemental compositions of PDA films and corresponding nanoparticles generated in the presence of $\mathrm{Na}^{+}$, $\mathrm{Mg}^{2+}, \mathrm{Ca}^{2+}, \mathrm{Co}^{2+}$, compared with pure PDA. The measured values of metallic ions in PDA films through XPS characterization were lower than that in nanoparticles, may be because metallic ions were trapped inside films deeply. These values of metallic ions in PDA films and nanoparticles obtained by ICPOES were similar (Table S2 $\dagger$ ). On the other hand, in all types of PDA, the contents of $\mathrm{N}$ and $\mathrm{O}$ in nanoparticles were higher than that in films. It is reported that the oxygen-rich region at air-water interface significantly enhances the crosslinking of

Table 1 Atomic ratios of $\mathrm{C}, \mathrm{N}, \mathrm{O}$ and metal ions in PDA films and corresponding nanoparticles (NPs) generated in the presence of $\mathrm{Na}^{+}$, $\mathrm{Mg}^{2+}, \mathrm{Ca}^{2+}, \mathrm{Co}^{2+}$ cations compared with that of pure PDA, measured by XPS

\begin{tabular}{|c|c|c|c|c|c|c|c|c|}
\hline \multirow{2}{*}{$\begin{array}{l}\text { Composition } \\
\text { at } \%\end{array}$} & \multicolumn{2}{|l|}{ C $1 \mathrm{~s}$} & \multicolumn{2}{|l|}{ N 1s } & \multicolumn{2}{|l|}{ O $1 \mathrm{~s}$} & \multicolumn{2}{|c|}{ Metal ions } \\
\hline & Film & NPs & Film & NPs & Film & NPs & Film & NPs \\
\hline PDA & 77.11 & 69.53 & 5.78 & 8.61 & 17.11 & 21.86 & - & - \\
\hline Na-PDA & 73.51 & 68.50 & 6.57 & 8.41 & 19.91 & 22.81 & - & 0.28 \\
\hline Mg-PDA & 72.46 & 69.27 & 7.55 & 8.54 & 19.99 & 21.88 & - & 0.31 \\
\hline Ca-PDA & 70.88 & 66.46 & 8.29 & 9.15 & 20.50 & 23.30 & 0.33 & 1.09 \\
\hline Co-PDA & 74.12 & 66.16 & 5.9 & 8.98 & 19.67 & 23.52 & 0.30 & 1.35 \\
\hline
\end{tabular}

PDA. $^{2}$ This crosslinking may induce the loss of some functional groups on PDA films, resulting in the lower contents of $\mathrm{N}$ and $\mathrm{O}$ in PDA films. The high-resolution XPS spectra of the C 1s, N 1s and $\mathrm{O}$ 1s core-level for Na-PDA, Mg-PDA, Ca-PDA and Co-PDA film samples were compared with neat PDA films (Fig. S6$\mathrm{S} 8 \dagger)$. Table $\mathrm{S} 3-\mathrm{S} 5 \dagger$ presented the detailed components in $\mathrm{C}$ $1 \mathrm{~s}, \mathrm{~N} 1 \mathrm{~s}$ and $\mathrm{O}$ 1s of PDA films and nanoparticles generated in different conditions. The similar compositions of PDA films and corresponding nanoparticles indicated that the PDA in films shared the same origin with those nanoparticles in solutions.

Since the upper and lower surfaces of PDA films were exposed to air or solutions, respectively, and their morphologies were significantly different, it would be interesting to know if their surface properties were similar or not. We have further measured the water contact angle of upper and lower surfaces of these PDA films (Fig. 5). All upper surfaces of PDA films presented a larger water contact angle than their lower surfaces showed. The values for the upper surfaces of PDA, Na-PDA, MgPDA and Ca-PDA were larger or near $90^{\circ}$, but the values of their corresponding lower surfaces were about $75 \pm 2^{\circ}$, slightly higher than the commonly reported values of PDA coatings (60$\left.70^{\circ}\right) .{ }^{24}$ In the surface modification using PDA, solid substrates were generally dipped in dopamine solution for PDA coating, so that the PDA coating on substrates showed similar surface property with the lower surfaces of our free-standing PDA films. The more hydrophobic upper surface may be also the result of higher crosslinking degree of PDA. Interestingly, the Co-PDA film was the most hydrophilic one in both upper and lower surfaces among all PDA films, which may be the result of the porous film body and the special in their functional groups.

Besides, the zeta-potential values of PDA nanoparticles obtained after 1 week reaction in the presence of different metallic 


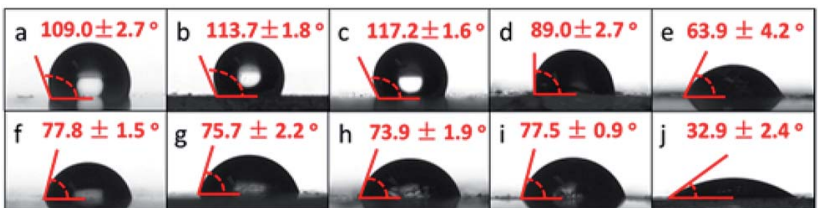

Fig. 5 The water contact angles of the upper surfaces and lower surfaces of polydopamine films generated in the presence of different metallic ions after 24 hours reaction. (a-e) The upper surfaces. $(f-j)$ The lower surfaces. PDA ( $a$ and $f) ; \mathrm{Na}$-PDA ( $b$ and $g$ ), $M g-P D A ~(c$ and $h$ ); Ca-PDA ( $d$ and i); Co-PDA (e and j).

ions were measured (Fig. 6). Compared with neat PDA, all PDA nanoparticles generated in salty conditions showed a decrease of their isoelectric point, from $\sim 4$ for pure PDA to 3.7 for NaPDA, 3.4 for Mg-PDA and Ca-PDA and 3.1 for Co-PDA. The difference should be caused by the change of functional groups on PDA obtained in different conditions. ${ }^{25}$ Previous study demonstrated that paramagnetic metal ions enhanced the rate of production of hydrogen peroxide during autoxidation of melanin pigments. ${ }^{26}$ The increased $\mathrm{H}_{2} \mathrm{O}_{2}$ under the environment with metallic ions will promote the oxidation of DHI to pyrrole carboxylic acid. ${ }^{27}$ The formation of pyrrole carboxylic acid was responsible for the lower zeta potential in $\mathrm{CaCl}_{2}$ oxidized PDA films. ${ }^{21}$ Thus the lower zeta potential of PDA nanoparticles fabricated in salty conditions is because they contain higher amount of pyrrole carboxylic acid than neat PDA has. This was supported by Raman spectra of the lower surfaces of PDA films, in which the peak at $1395 \mathrm{~cm}^{-1}$, representing the stretching of pyrrole ring, was pronounced in PDA generated in salty conditions (Fig. S9†). Moreover, the lowest isoelectric point of Co-PDA nanoparticles is agreed with the most hydrophilic nature of Co-PDA films, showing that Co-PDA contains more pyrrole carboxylic acid, the downstream product of PDA oxidation.

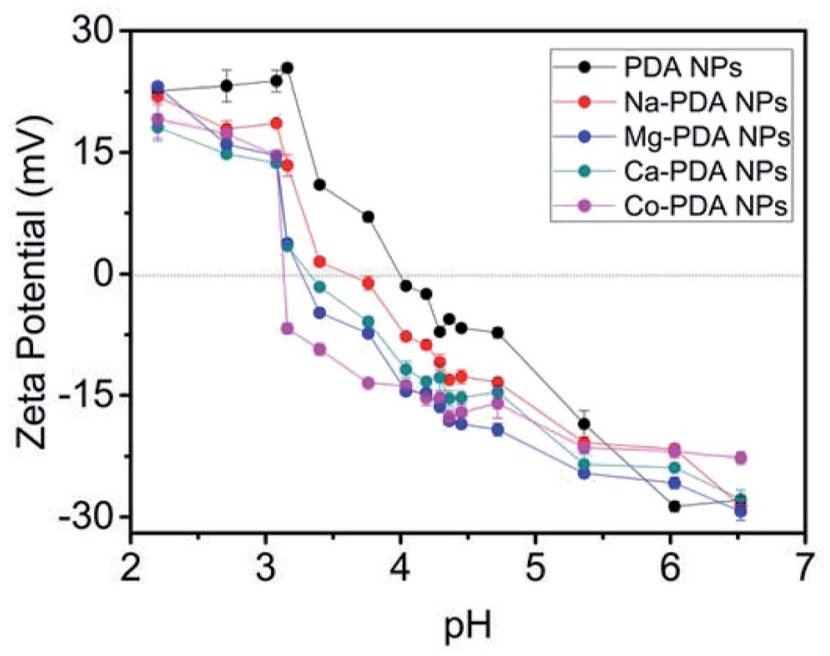

Fig. 6 Zeta potential of PDA nanoparticles obtained after 1 week reaction in the presence of different metal ions. The concentrations of adding $\mathrm{NaCl}, \mathrm{MgCl}_{2}, \mathrm{CaCl}_{2}$ and $\mathrm{CoCl}_{2}$ were $100 \mathrm{mM}$.

\section{Discussion}

Because of the mixture nature of PDA, it is hard to clearly identify the different chemical compositions in Na-PDA, CaPDA, Mg-PDA and Co-PDA, although their nanoparticles demonstrated difference in surface charge. However, based on their absorption spectra, we can identify that in the initial stage the intermediates in oxidative polymerization of dopamine changed in different conditions. There are three different intermediates clearly observed in these systems: (1) in neat dopamine, Na-dopamine and Ca-dopamine cases, DQ mixed with AC in the initial stage; (2) only AC in Co-dopamine; and (3) $\mathrm{AC}$ and sequinone-dimer in Mg-dopamine. In case of Codopamine, $\mathrm{Co}$ (II) can form complex with AC (Scheme 1), the fast ligand exchange in solutions lead the AC being dominated in the UV-vis spectra. On the other hand, based on theoretical calculations, ${ }^{28,29}$ forming complex of $o$-semiquinone radical ${ }^{28}$ is possible in both $\mathrm{Ca}^{2+}$ and $\mathrm{Mg}^{2+}$ cases, but we could only identify it clearly in Mg-dopamine system, which was because the lifetime of the complex of $\mathrm{Mg}$ with semiquinone radicals was much longer than that of $\mathrm{Ca}(\mathrm{II})$ complex. ${ }^{29}$ The purple colour in $\mathrm{Mg}$ system decayed after a quite long reaction time ( $>1 \mathrm{~h})$, showing a good stability of $\mathrm{Mg}$ (II)-semiquinone-dimer intermediate. The long-time lasting of purple colour has reported in the oxidative polymerization of 5,6-dihydroxyindole (DHI) in chicken egg white $^{30}$ or DHICA in PVA solution. ${ }^{31}$ The tautomeric forms of quionones from DHI dimers under pulse radiation also presented absorptions around $500-600 \mathrm{~nm}$ based on theoretical studies. ${ }^{23}$ This proposed dimer complex (Scheme 1) is distinguished with the well-known coordination effect of $\mathrm{Fe}(\mathrm{IIII})^{32}$ and $\mathrm{Ni}(\mathrm{II})^{33}$ via catechol, oxidation of $\mathrm{Cu}(\mathrm{II})$ and $\mathrm{Ce}(\mathrm{IV}){ }^{34,35}$ chargetransfer effect of $\mathrm{Ti}(\mathrm{Iv}),{ }^{36}$ and ionic strength of $\mathrm{Na}(\mathrm{I}) \cdot{ }^{37}$ It indicated that there were various pathways of metallic ions involving in dopamine polymerization.

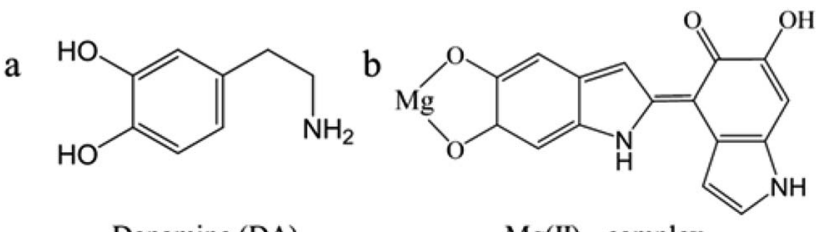

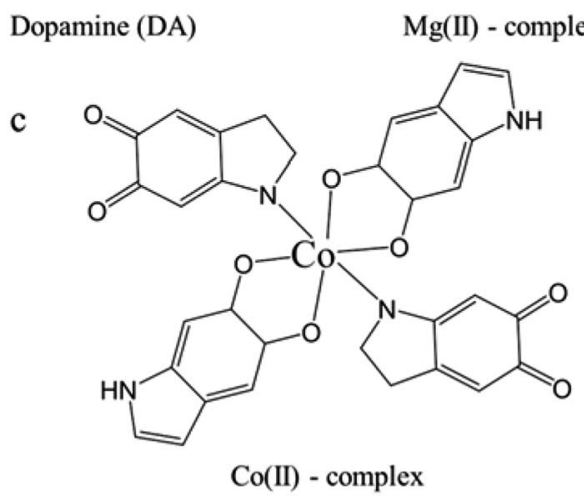

Scheme 1 The chemical structures of dopamine (a), the proposed complex composed of $\mathrm{Mg}(\|)$ and semiquinone dimer (b), and the proposed complex composed of Co(II), semiquinone and aminochrome (c). ${ }^{38}$ 
On the other hand, characterization results presented useful information for understanding the formation mechanism of PDA film. Fortini et al. studied the drying of films containing mixtures of large and small colloidal particles in water. ${ }^{39}$ They observed that the mixture stratified into a layer of the larger particles at the bottom with a layer of the smaller particles on the top due to the gradient of osmotic pressure in drying. ${ }^{39}$ Considering similar composition and different sizes of PDA nanoparticles composed of films and suspensions (Fig. S10 $\dagger$ ), we suppose that the formation of PDA films may also the result of the stratification of finer PDA nanoparticles in solutions (Scheme 2). In the oxidative polymerization of dopamine, active oxygen free radicals work as the oxidant. There is more oxygen in solution near the air-solution interface, the oxidative polymerization started in this region. The presence of different metallic ions influences the speed of oxidative polymerization, and in turn the size distribution of PDA colloids in different conditions. It is observed that a large size difference and high volume fraction of small particles enhanced the degree of stratification, with small particles favoured to be at the surface, ${ }^{\mathbf{4 0}}$ and facilitated diffusion of small nanoparticles into the interstitial spaces among particles. ${ }^{\mathbf{4 1}}$ In initial stage, due to the stratification, finer nanoparticles were aggregated as PDA films as shown in AFM characterization (Fig. 4). In the middle of reaction period, PDA nanoparticles with broad size-distribution gathered in solution, but still those smaller particles were packed in films. The polymerization in the solution trapped between PDA nanoparticles on films resulted in infilling of these interstitial spaces, giving smooth upper surface of PDA
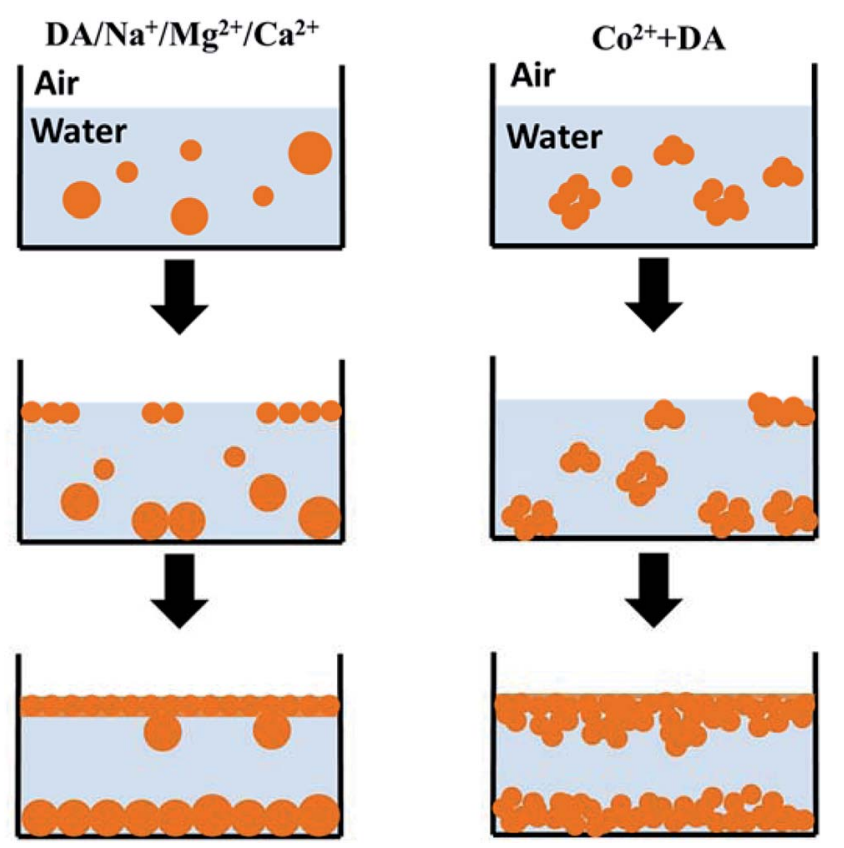

Scheme 2 Schematic illustration of two different stratification processes demonstrated in PDA systems. In neat PDA and that with $\mathrm{Na}^{+}, \mathrm{Ca}^{2+}$ and $\mathrm{Mg}^{2+}$, stratification leads to the aggregation of finer PDA nanoparticles at the air-solution interface, giving smooth surface and solid film body; whereas fast polymerization in Co-PDA system results in the aggregation of PDA nanoaggregates at interface, resulting in Co-PDA film with rugged surface and porous film body.

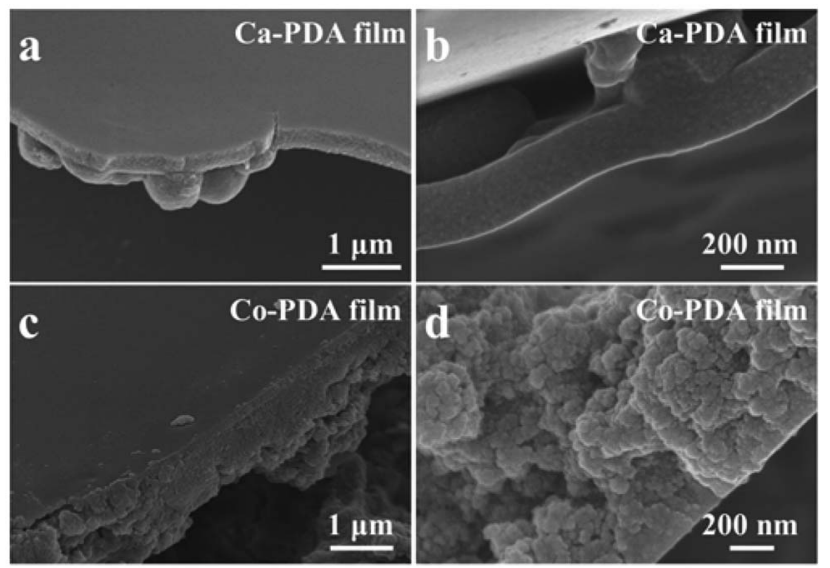

Fig. 7 SEM images of the two identical morphologies of PDA films. Ca-PDA film has smooth upper surface and solid film body (a and b), while Co-PDA film with rugged surface and porous film body ( $c$ and $d$ ).

films. In the late stage of polymerization, there was full of large aggregates or large particles in solutions with decreased number of small PDA nanoparticles, thus some large particles were trapped in the lower surface of PDA films by Brownian motion. The film following above formation process has smooth surface and solid film body (as Ca-PDA in Fig. 7). The case of Co-dopamine was different. As we observed in UV-vis spectra, the aggregation of PDA nanoparticles in Co-dopamine happened much faster than that in other systems. There was no finer and isolated PDA nanoparticles even in the early stage of stratification, only aggregates of PDA nanoparticles (Fig. 4e), thus the Co-PDA film was rugged. The infilling due to further polymerization of solutions smoothed this film slightly, but it cannot be as compact as other PDA films, giving a porous film body (Fig. 7).

\section{Conclusions}

In this study, we compared the oxidative polymerization of dopamine in the presence of different metallic ions, $\mathrm{Na}^{+}, \mathrm{Ca}^{2+}$, $\mathrm{Mg}^{2+}$ and $\mathrm{Co}^{2+}$, mainly focusing on the relations between their free-standing films generated at the air-solution interface and corresponding nanoparticles in solutions. UV-vis characterizations presented different intermediates involving in the dopamine polymerization in various conditions. The complex of $\mathrm{Mg}$ (II) with semiquinone dimer is distinct from the coordination between dopamine and other metallic cations. On the basis of comparative analysis of morphologies and chemical compositions of PDA films and their corresponding nanoparticles, we proposed a stratification-based mechanism for PDA films generated in different systems. These investigations enrich our knowledge of dopamine polymerization in various environments, and guide us in the further application of freestanding PDA films.

\section{Conflicts of interest}

There are no conflicts to declare. 


\section{Acknowledgements}

The authors acknowledge financial support from the National Natural Science Foundation of China (Grant 51673210).

\section{Notes and references}

1 F. Ponzio, P. Payamyar, A. Schneider, M. Winterhalter, J. Bour, F. Addiego, M.-P. Krafft, J. Hemmerle and V. Ball, J. Phys. Chem. Lett., 2014, 5, 3436-3440.

2 S. Hong, C. F. Schaber, K. Dening, E. Appel, S. N. Gorb and H. Lee, Adv. Mater., 2014, 26, 7581-7587.

3 T.-F. Wu and J.-D. Hong, Biomacromolecules, 2015, 16, 660666.

4 T.-F. Wu, B.-H. Wee and J.-D. Hong, Adv. Mater. Interfaces, 2015, 2, 1500203.

5 V. Ball, D. D. Frari, M. Michel, M. J. Buehler, V. Toniazzo, M. K. Singh, J. Gracio and D. Ruch, Bionanoscience, 2011, 2, 16-34.

6 F. Ponzio and V. Ball, Polym. Int., 2016, 65, 1251-1257.

7 M. Salomäki, M. Tupala, T. Parviainen, J. Leiro, M. Karonen and J. Lukkari, Langmuir, 2016, 32, 4103-4112.

8 T. G. Barclay, H. M. Hegab, S. R. Clarke and M. GinicMarkovic, Adv. Mater. Interfaces, 2017, 4, 1601192.

9 H.-C. Yang, W. Xu, Y. Du, J. Wu and Z.-K. Xu, RSC Adv., 2014, 4, 45415-45418.

10 J. H. Ryu, P. B. Messersmith and H. Lee, ACS Appl. Mater. Interfaces, 2018, 10, 7523-7540.

11 M. J. Harrington, A. Masic, N. Holten-Andersen, J. H. Waite and P. Fratzl, Science, 2010, 328, 216-220.

12 Z. P. Xu, Sci. Rep., 2013, 3, 2914.

13 E. Degtyar, M. J. Harrington, Y. Politi and P. Fratzl, Angew. Chem., Int. Ed., 2014, 53, 12026-12044.

14 K. Chen, J. Ding, S. H. Zhang, X. K. Tang, Y. H. Yue and L. Guo, ACS Nano, 2017, 11, 2835-2845.

15 N. Holten-Andersen, T. E. Mates, M. S. Toprak, G. D. Stucky, F. W. Zok and J. H. Waite, Langmuir, 2009, 25, 3323-3326.

16 J. J. Wilker, Curr. Opin. Chem. Biol., 2010, 14, 276-283.

17 J. Yang, M. A. Cohen Stuart and M. Kamperman, Chem. Soc. Rev., 2014, 43, 8271-8298.

18 H. B. Zeng, D. S. Hwang, J. N. Israelachvili and J. H. Waite, Proc. Natl. Acad. Sci. U. S. A., 2010, 107, 12850-12853.

19 D. S. Hwang, H. B. Zeng, A. Masic, M. J. Harrington, J. N. Israelachvili and J. H. Waite, J. Biol. Chem., 2010, 285, 25850-25858.

20 F. Byette, A. Laventure, I. Marcotte and C. Pellerin, Biomacromolecules, 2016, 17, 3277-3286.

21 L. Klosterman and C. J. Bettinger, Int. J. Mol. Sci., 2017, 18, 14.
22 M. Bisaglia, S. Mammi and L. Bubacco, J. Biol. Chem., 2007, 282, 15597-15605.

23 A. Pezzella, L. Panzella, O. Crescenzi, A. Napolitano, S. Navaratman, R. Edge, E. J. Land, V. Barone and M. d'Ischia, J. Am. Chem. Soc., 2006, 128, 15490-15498.

24 H. Lee, S. M. Dellatore, W. M. Miller and P. B. Messersmith, Science, 2007, 318, 426-430.

25 W. C. Zheng, H. L. Fan, L. Wang and Z. X. Jin, Langmuir, 2015, 31, 11671-11677.

26 P. Hintz and B. Kalyanaraman, Biochim. Biophys. Acta, Gen. Subj., 1986, 883, 41-45.

27 B. Szpoganicz, S. Gidanian, P. Kong and P. Farmer, J. Inorg. Biochem., 2002, 89, 45-53.

28 M. Witwicki and J. Jezierska, J. Phys. Chem. B, 2011, 115, 3172-3184.

29 M. Witwicki and J. Jezierska, Theor. Chem. Acc., 2013, 132, 1383.

30 N. F. della Vecchia, P. Cerruti, G. Gentile, M. E. Errico, V. Ambrogi, G. D'Errico, S. Longobardi, A. Napolitano, L. Paduano, C. Carfagna and M. d'Ischia, Biomacromolecules, 2014, 15, 3811-3816.

31 R. Micillo, L. Panzella, M. Iacomino, G. Prampolini, I. Cacelli, A. Ferretti, O. Crescenzi, K. Koike, A. Napolitano and M. d'Ischia, Sci. Rep., 2017, 7, 41532.

32 M. J. Sever, J. T. Weisser, J. Monahan, S. Srinivasan and J. J. Wilker, Angew. Chem., Int. Ed., 2004, 43, 448-450.

33 L. P. Yang, J. H. Kong, D. Zhou, J. M. Ang, S. L. Phua, W. A. Yee, H. Liu, Y. Z. Huang and X. H. Lu, Chem.-Eur. J., 2014, 20, 7776-7783.

34 F. Bernsmann, V. Ball, F. Addiego, A. Ponche, M. Michel, J. J. de Almeida Gracio, J. J. Gracio, V. Toniazzo and D. Ruch, Langmuir, 2011, 27, 2819-2825.

35 A. Hayat, D. Andreescu, G. Bulbul and S. Andreescu, J. Colloid Interface Sci., 2014, 418, 240-245.

36 G. Vitiello, A. Pezzella, A. Zanfardino, M. Varcamonti, B. Silvestri, A. Costantini, F. Branda and G. Luciani, J. Mater. Chem. B, 2015, 3, 2808-2815.

37 J. H. Kuang, J. L. Guo and P. B. Messersmith, Adv. Mater. Interfaces, 2014, 1, 1400145.

38 D. Sato, Y. Shiota, G. Juhász and K. Yoshizawa, J. Phys. Chem. A, 2010, 114, 12928-12935.

39 A. Fortini, I. Martin-Fabiani, J. L. De La Haye, P. Y. Dugas, M. Lansalot, F. D'Agosto, E. Bourgeat-Lami, J. L. Keddie and R. P. Sear, Phys. Rev. Lett., 2016, 116, 118301.

40 J. J. Zhou, Y. Jiang and M. Doi, Phys. Rev. Lett., 2017, 118, 108002.

41 H. Luo, C. M. Cardinal, L. E. Scriven and L. F. Francis, Langmuir, 2008, 24, 5552-5561. 